\title{
miR-203 inhibits the migration and invasion of esophageal squamous cell carcinoma by regulating LASP1
}

\author{
NOBUYOSHI TAKESHITA, MIKITO MORI, MASAYUKI KANO, ISAMU HOSHINO, YASUNORI AKUTSU, \\ NAOYUKI HANARI, YASUO YONEYAMA, NORIMASA IKEDA, YUKA ISOZAKI, TETSUROU MARUYAMA, \\ NAOKI AKANUMA, YUKIMASA MIYAZAWA and HISAHIRO MATSUBARA
}

Department of Frontier Surgery, Chiba University Graduate School of Medicine, Chiba, Japan

Received June 24, 2012; Accepted August 6, 2012

DOI: $10.3892 /$ ijo.2012.1614

\begin{abstract}
The expression of microRNA-203 (miR-203) in esophageal squamous cell carcinoma (ESCC) tissues is remarkably lower than that in non-ESCC tissues. We investigated how miR-203 could influence the development of ESCC cells. Our analyses revealed that miR-203 inhibited the migration and invasion of ESCC cells. Genome-wide gene expression data and target site inhibition assays showed that miR-203 appears to directly regulate LIM and SH3 protein 1 (LASP1). The knockdown of LASP1 resulted in inhibition of the migration and invasion of ESCC cells. Our results suggest that miR-203 and its target LASP1, may be associated with the progression of ESCC. In clinical ESCC specimens, the expression levels of miR-203, which were lower compared to those in normal tissues, were inversely correlated with the mRNA expression levels of LASP1. Moreover, we found that there was a significant correlation between the expression levels of miR-203 and the relapse-free survival. The identification of a cancer network regulated by miR-203 could provide new insights into the potential mechanisms of the progression of ESCC.
\end{abstract}

\section{Introduction}

Esophageal squamous cell carcinoma (ESCC) is known to have one of the worst prognoses of any cancers worldwide, and the overall 5-year survival rate of ESCC is currently $25-30 \%(1,2)$. Recent advances in the multidisciplinary diagnostic and therapeutic approaches have improved the prognosis of the patients with $\operatorname{ESCC}(3,4)$, but the prognosis of these patients is still poor compared to that of the patients with breast and colon cancer $(5,6)$. The elucidation of the molecular features of ESCC cells could contribute to improvements in the diagnosis and treatment of patients with ESCC.

Correspondence to: Dr Mikito Mori, Department of Frontier Surgery, Chiba University Graduate School of Medicine, Chiba University, Inohana 1-8-1, Chuo-ku, Chiba 260-8670, Japan

E-mail: mikimori@faculty.chiba-u.jp

Key words: microRNA, miR-203, esophageal squamous cell carcinoma, LASP1
MicroRNA (miRNA) is regarded to be one of the most practical biological approaches to investigate the causative mechanisms of various diseases. It has been suggested that miRNA can suppress the expression of target genes by repressing mRNA translation or cleaving the target mRNA, and miRNAs are thought to regulate a wide range of biological phenomena, such as cell growth, proliferation, differentiation, and death (7-10). Therefore, it has been supposed that aberrations in the expression of miRNAs might affect the development of various malignancies by altering the expression of oncogenes and tumor suppressor genes (11-14), and several studies have showed that identifying a unique subset of miRNAs that are differentially expressed in tumors might contribute to improvements in the diagnosis and treatment of various types of cancers $(15,16)$. A few genome-wide miRNA expression studies of ESCC have been published, including our previous report, and some promising miRNAs for the identification and/or treatment of ESCC have been identified (17-20), but the biological behavior of miRNAs in ESCC cells remains unclear. Therefore, further investigations are needed to clarify the role of miRNAs in the development of ESCC.

We previously identified a novel subset of 15 downregulated miRNAs by our comprehensive miRNA profiling study of ESCC specimens. In this study, we analyzed the effects of miR-203, which was one of the 15 downregulated miRNAs, on ESCC cell lines using proliferation, migration, and invasion assays. Of the candidate target genes of miR-203, we selected LIM and SH3 protein 1 (LASP1) for a further analysis, because it was reported that LASP1 is involved in breast and colorectal cancer metastasis $(21,22)$, and verified that LASP1 was directly regulated by miR-203 in ESCC. Furthermore, the expression of miR-203 was found to be inversely correlated with the LASP1 expression in ESCC specimens. The finding of the association between miR-203 and LASP1 might assist in understanding the molecular mechanism of the progression of ESCC.

\section{Materials and methods}

Clinical ESCC specimens and ESCC cell culture. Eighteen pairs of primary esophageal squamous cell carcinoma and corresponding normal esophageal epithelia tissue sections were obtained from patients who had undergone surgery at Chiba University Hospital from 2004 to 2005 . All tissue specimens 
were obtained from previously untreated patients undergoing primary surgical treatment. Normal tissues were obtained far from the center of the cancer from surgical specimens. No cancer cells were detected in neighboring formalin-fixed paraffin-embedded (FFPE) specimens. Written consent for tissue donation for research purposes was obtained from each patient before tissue collection. The protocol was approved by the Institutional Review Board of Chiba University. The specimens were snap-frozen in liquid nitrogen and stored at $-80^{\circ} \mathrm{C}$.

TE2 cells, a squamous cancer cell line with wild-type p53 derived from human ESCC, were provided by the Department of Surgery, Tohoku University School of Medicine. T.Tn cells, from a human ESCC cell line known to contain an oncogenic p53 mutation, were provided by the Japanese Cancer Research Resources Bank. These cell lines have been tested and authenticated as described in the literature $(23,24)$. Both cell lines were cultured in Dulbecco's modified Eagle's medium nutrient mixture (DMEM; Life Technologies, Grand Island, NY) supplemented with $10 \%$ fetal bovine serum (FBS) in a humidified atmosphere containing $5 \% \mathrm{CO}_{2}$ at $37^{\circ} \mathrm{C}$.

RNA isolation. Tissues and cells were treated with the TRIzol reagent (Invitrogen, Carlsbad, CA, USA), according to the manufacturer's instructions, for total-RNA extraction. The extracted RNA was quantified by absorbance at $260 \mathrm{~nm}$, and its purity was evaluated by examining the 260/280 ratio of absorbance using a SmartSpec ${ }^{\mathrm{TM}}$ Plus spectrophotometer (Bio-Rad Laboratories, Hercules, CA, USA).

Mature miRNA transfection and small interfering RNA (siRNA) treatment. Mature miRNA molecules, pre-miR ${ }^{\mathrm{TM}}$ miRNA precursors (hsa-miR-203; Pre-miR ID: PM10152 and negative control miRNA; P/N: AM17110) (Applied Biosystems, Foster City, CA, USA) were incubated with Opti-MEM (Invitrogen) and the siPORT ${ }^{\mathrm{TM}} \mathrm{NeoFX}^{\mathrm{TM}}$ transfection reagent (Invitrogen).

The small interfering RNA (Silencer ${ }^{\circledR}$ Select Pre-designed siRNA; LASP1 Cat. no. 4392420) and negative control siRNA (Silencer ${ }^{\circledR}$ Select Negative Control no. 1 siRNA Cat. no. 4390843) (Ambion) were incubated with Opti-MEM (Invitrogen) and the Lipofectamine $^{\mathrm{TM}}$ RNAiMax Reagent (Invitrogen).

As recommended by the manufacturer, we first tested the transfection efficacy of miRNA into the ESCC cell lines based on the downregulation of protein tyrosine kinase 9 (PTK9) mRNA after transfection with miR-1.

Cell proliferation, migration, and invasion assays in ESCC cell lines. Cells were reverse transfected with $10 \mathrm{nM}$ miRNA or si-LASP1 and plated in 96-well plates at $3 \times 10^{3}$ cells per well in $100 \mu \mathrm{l}$ of medium containing $10 \%$ FBS. After $72 \mathrm{~h}$, cell proliferation was assessed with the Cell Counting Kit-8 (Dojindo, Kumamoto, Japan). Triplicate wells were measured for cell viability in each treatment group.

Cell motility was determined using a micropore chamber assay; $8 \times 10^{4}$ cells were seeded onto the top chamber of a 24-well micropore polycarbonate membrane filter with $8 \mu \mathrm{m}$ pores (BD Biosciences, Bedford, MA, USA) and the bottom chamber was filled with DMEM containing 10\% FBS as a chemoattractant. After $24 \mathrm{~h}$ of incubation, the membranes were fixed and stained by the DiffQuik reagent (International
Reagents, Kobe, Japan) and the cells on the upper surface were carefully removed with a cotton swab. Cell migration was quantified by counting the average number of migrated cells in 4 random high-powered fields per filter. For the assessment of cell invasion, the migration assay was repeated with a 24-well Matrigel-coated micropore membrane filter with $8 \mu \mathrm{m}$ pores (BD Biosciences). Cell invasion was quantified by counting the number of cells that had migrated through the membrane.

Screening for miR-203 target genes by a microarray analysis. A genome-wide screen was performed for identify the genes silenced by miR-203 in ESCC cells (TE2 and T.Tn). GeneChip U133 Plus 2.0 Arrays (Affymetrix, Santa Clara, CA, USA) were used for the expression profiling of miR-203 transfectants in comparison with miRNA-negative control transfectants in the ESCC cell lines. Starting with $250 \mathrm{ng}$ of total-RNA, the GeneChip U133 Plus 2.0 Array was conducted in accordance with manufacturer's protocol, and using the GeneChip 3'IVT Express Kit (Affymetrix) and GeneChip Hybridization Wash and Stain Kit (Affymetrix). The raw expression signal obtained from the GeneChip Instrument System (Affymetrix) was normalized by scaling to the target signal of 100 .

The predicted target genes and their conserved sites where the seed region of each miRNA binds were investigated using the TargetScan database (release 5.1, http://www.targetscan. org/). The sequence of the predicted mature microRNA was confirmed using the miRBase software program (release 17.0, http://microrna.sanger.ac.uk/).

Quantitative reverse-transcription-PCR ( $q R T-P C R)$. Firststrand cDNA was synthesized from $1.0 \mu \mathrm{g}$ of total-RNA using a High Capacity cDNA Reverse Transcription kit (Applied Biosystems). Gene-specific PCR products were assayed continuously using a 7300 real-time PCR system (Applied Biosystems) according to the manufacturer's protocol. TaqMan ${ }^{\circledR}$ probes and primers for LASP1 $(\mathrm{P} / \mathrm{N}$ : Hs01078815_m1) and glyceraldehyde-3-phosphate dehydrogenase (GAPDH) (P/N: Hs02758991_g1) as an internal control were obtained from Applied Biosystems (Assay-OnDemand Gene Expression Products). The expression levels of miR-203 (assay ID: 000507) were analyzed by TaqMan quantitative real-time PCR (TaqMan MicroRNA Assay, Applied Biosystems) and normalized to the level of RNU6B (assay ID: 001093). All reactions were performed in triplicate, and included a negative control that lacked cDNA.

Western blot analysis. The cells were harvested and lysed $72 \mathrm{~h}$ after transfection. Protein extracts $(40 \mu \mathrm{g})$ were separated by sodium dodecyl sulfate-polyacrylamide gel electrophoresis and transferred to polyvinylidene difluoride membranes. Membranes were blocked in PBS containing 5\% non-fat dried milk and $0.1 \%$ Tween-20. The anti-human LASP1 rabbit polyclonal $\mathrm{IgG}$ was used at a dilution of 1:100 and the anti-rabbit IgG-peroxidase antibody produced in a goat (Sigma-Aldrich, St. Louis, MO, USA) was used at a dilution of 1:5,000. The anti- $\beta$-actin polyclonal antibody (Santa Cruz Biotechnology) at a dilution of 1:200 and anti-goat IgG-peroxidase antibody produced in rabbits (Sigma-Aldrich) at a dilution of 1:5,000 were used as controls. After incubation with a primary 

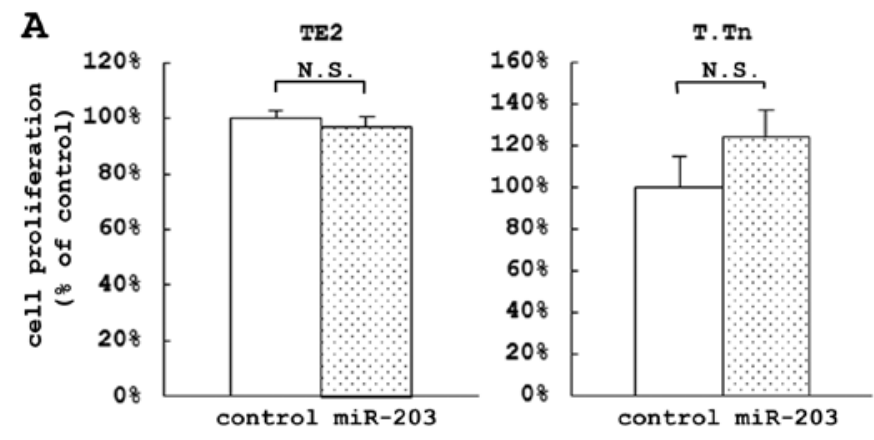

B

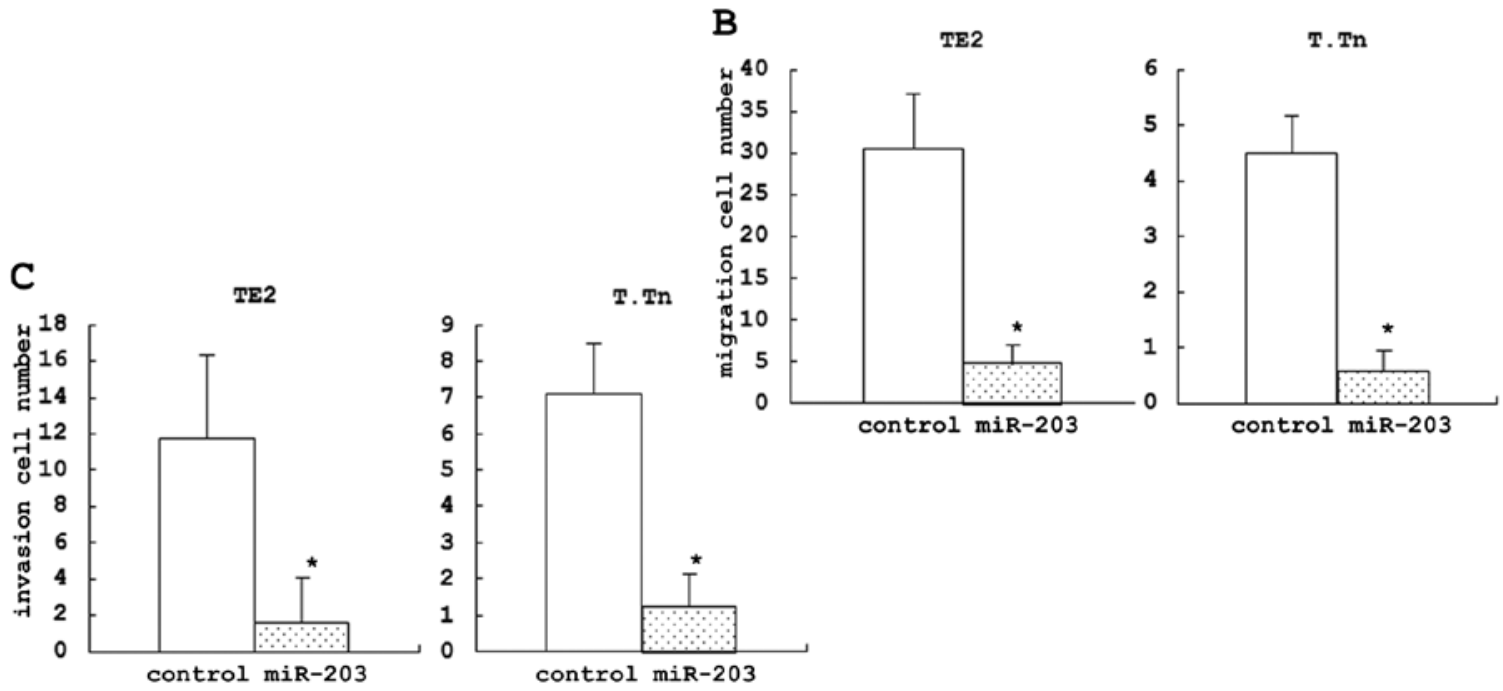

Figure 1. The effects of miR-203 knockdown on ESCC cell proliferation, migration and invasion. ESCC cell lines (TE2 and T.Tn) were transfected with 10 nM of miR-203 or control miRNA for $72 \mathrm{~h}$, then were compared to control cells for their (A) proliferation, (B) migration and (C) invasion. ${ }^{*} \mathrm{P}<0.05$.

antibody for $2 \mathrm{~h}$ at room temperature, the membranes were incubated for $1 \mathrm{~h}$ with the secondary antibody, also at room temperature. The analysis was performed using ECL Western blotting detection reagents (Amersham Pharmacia Biotech, Piscataway, NJ, USA). The expression levels of these proteins were evaluated by ImageJ software (version 1.44; http://rsbweb. nih.gov/ij/index.html).

Target site inhibition assays. The miScript Target Protector for the miR-203 binding site in the 3'UTR of LASP1 mRNA was obtained from Qiagen (target binding site sequence provided: 5'-GAAAUUUGCCUCUGUCCAAACAUUUCAUCC-3'). The miScript Target Protector is single-stranded, modified RNA that specially interferes with the interaction of a miRNA with a single target, while leaving the regulation of other targets of the same miRNA unaffected.Syn-hsa-miR-203 miScript miRNA Mimic (Qiagen, miScript miRNA Mimic for: GUGAAAUGU UUAGGACCACUAG) and miScript Target Protector were co-transfected into ESCC cell lines (TE2 and T.Tn) according to the manufacturer's protocol. Syn-hsa-miR-203 miScript miRNA Mimic and Negative control miScript Target Protector designed not to bind the mRNA of mammals were co-transfected into ESCC cell lines as a negative control. After $48 \mathrm{~h}$ of transfection, the total-RNA was isolated and the mRNA expression levels of LASP1 were measured by TaqMan quantitative real-time PCR.
Statistical analysis. The relationship between two variables and numerical values obtained by real-time RT-PCR were analyzed using the Mann-Whitney U test or paired t-test. The relationships between miR-203 expression and LASP1 expression were analyzed using the Spearman rank correlation. To evaluate the significance of the expression of miR-203 as a prognostic factor, we performed an analysis using the Kaplan-Meier method and the log-rank test. The Expert StatView software program (version 5, SAS Institute Inc., Cary, NC, USA) was used for the analysis, with statistical significance defined as $\mathrm{P}<0.05$.

\section{Results}

The effects of miRNA transfection on ESCC cell lines. The expression levels of miR-203 were extremely low in ESCC cell lines (data not shown), suggesting that there was no effect of the corresponding endogenous miR-203 on the viability of these cell lines. In a gain-of-function analysis, two cell lines (TE2 and T.Tn) were transfected with miR-203.

The proliferation assay, which was used to evaluate the proportion of cell growth inhibition of the cells transfected with miR-203 compared to the control cells at $72 \mathrm{~h}$ after the transfection, indicated that there was no significant differences between the cells transfected with miR-203 and the control in both the TE2 and T.Tn cell lines (Fig. 1A). The proliferation assay did not show any significant difference in the cell growth inhibition at 24, 48 and $96 \mathrm{~h}$ (data not shown). 
Table I. Gene downregulated in miR-203-transfected ESCC cell lines compared to control cells, which were found to have conserved sites in their 3' untranslated region using the TargetScan database.

\begin{tabular}{|c|c|c|c|c|}
\hline No & $\begin{array}{l}\text { Entrez } \\
\text { gene ID }\end{array}$ & $\begin{array}{l}\text { Gene } \\
\text { symbol }\end{array}$ & Gene name & $\begin{array}{l}\text { miR-203 } \\
\text { target site }\end{array}$ \\
\hline 1 & 3927 & LASP1 & LIM and SH3 protein 1 & 1 \\
\hline 2 & 90161 & HS6ST2 & Heparan sulfate 6-O-sulfotransferase 2 & 1 \\
\hline 3 & 51496 & CTDSPL2 & $\begin{array}{l}\text { CTD (carboxy-terminal domain, RNA polymerase II, } \\
\text { polypeptide A) small phosphatase like } 2\end{array}$ & 1 \\
\hline 4 & 11333 & PDAP1 & PDGFA associated protein 1 & 2 \\
\hline 5 & 1352 & COX10 & $\begin{array}{l}\text { COX10 homolog, cytochrome } c \text { oxidase assembly protein, } \\
\text { heme A: farnesyltransferase }\end{array}$ & 1 \\
\hline 6 & 3600 & IL15 & Interleukin 15 & 1 \\
\hline 7 & 2186 & BPTF & Bromodomain PHD finger transcription factor & 1 \\
\hline 8 & 9037 & SEMA5A & $\begin{array}{l}\text { Sema domain, seven thrombospondin repeats (type } 1 \text { and } \\
\text { type } 1 \text {-like), transmembrane domain (TM) and } \\
\text { short cytoplasmic domain, (semaphorin) } 5 \mathrm{~A}\end{array}$ & 2 \\
\hline 9 & 27445 & PCLO & Piccolo (presynaptic cytomatrix protein) & 1 \\
\hline 10 & 54665 & RSBN1 & Round spermatid basic protein 1 & 1 \\
\hline 11 & 196528 & ARID2 & AT rich interactive domain 2 (ARID, RFX-like) & 1 \\
\hline 12 & 51714 & SELT & Selenoprotein $\mathrm{T}$ & 1 \\
\hline 13 & 3607 & FOXK2 & Forkhead box K2 & 1 \\
\hline 14 & 518 & ATP5G3 & $\begin{array}{l}\text { ATP synthase, H+ transporting, mitochondrial Fo complex, } \\
\text { subunit C3 (subunit 9) }\end{array}$ & 1 \\
\hline 15 & 55573 & CDV3 & CDV3 homolog & 1 \\
\hline 16 & 114882 & OSBPL8 & Oxysterol binding protein-like 8 & 1 \\
\hline 17 & 57507 & ZNF608 & Zinc finger protein 608 & 1 \\
\hline 18 & 54557 & SGTB & Small glutamine-rich tetratricopeptide repeat (TPR)-containing, $\beta$ & 1 \\
\hline 19 & 160897 & GPR180 & G protein-coupled receptor 180 & 1 \\
\hline 20 & 91749 & KIAA1919 & KIAA1919 & 1 \\
\hline 21 & 148867 & SLC30A7 & Solute carrier family 30 (zinc transporter), member 7 & 1 \\
\hline 22 & 55219 & TMEM57 & Tansmembrane protein 57 & 1 \\
\hline
\end{tabular}

In the migration assay, we evaluated the proportion of the inhibition of cell motility of the cells transfected with miR-203 compared to the control cells at $72 \mathrm{~h}$. After the transfection, we found a significant difference between these cell lines (84.2\% in TE2 and $87.0 \%$ in T.Tn) (Fig. 1B). The invasion assay was used to evaluate the proportion of the inhibition of cell invasion of the cells transfected with miR-203 compared to the control cells. Using this assay, we also found a significant difference between the miRNA and control-transfected cells at $72 \mathrm{~h}$ after the transfection $(86.5 \%$ in TE2 and $82.4 \%$ in T.Tn) (Fig. 1C).

LASPI as one of the direct target genes of miR-203. An analysis of the genome-wide gene expression of the target genes silenced by miR-203 was performed using a microarray analysis of the ESCC cells transfected with the miR-203 and the control. A total of 22 genes were identified to be downregulated, and we assessed the conserved sites in the $3^{\prime} \mathrm{UTR}$ using the TargetScan database (Table I). During the search of the TargetScan database, we found one conserved site in the 3'UTR of LASP1 (Fig. 2A).
The expression levels of LASP1 mRNA in the cells transfected with miR-203 were significantly lower than those in the control cells (25.9\% of the control in TE2 cells and $46.6 \%$ in the T.Tn cells) (Fig. 2B). After $72 \mathrm{~h}$, the protein expression levels were also reduced (Fig. 2C).

These two cell lines were also used to investigate how miR-203 directly affects LASP1 expression. After $48 \mathrm{~h}$ of co-transfection with Syn-hsa-miR-203 using a miScript miRNA Mimic and miScript Target Protector/Negative control miScript Target Protector, the expression levels of LASP1 mRNA in the transfected cells were significantly higher than those in the control cells $(22.3 \%$ of the control in TE2 cells and $49.2 \%$ in T.Tn cells). These results suggested that this specific target may be directly controlled by miR-203 (Fig. 2D).

Evaluation of the effects of LASP1 on the proliferation, migration, and invasion of ESCC cell lines using a loss of function analysis. After $48 \mathrm{~h}$ of si-LASP1 transfection in the two cell lines, we confirmed that the expression levels of LASP1 mRNA were remarkably decreased $(80.4 \%$ of the control in TE2 cells and 65.5\% in T.Tn cells) (Fig. 3A). We 

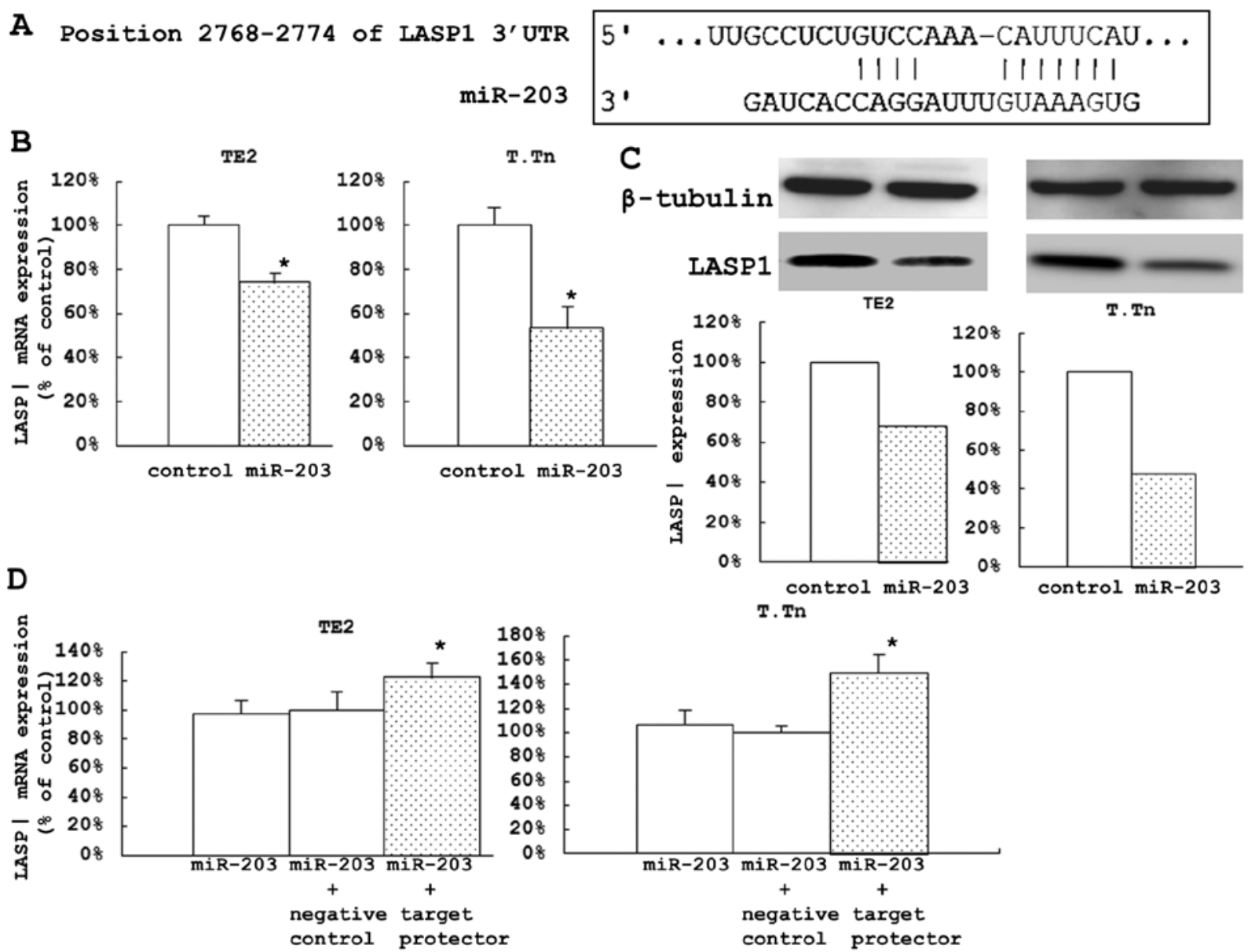

Figure 2. (A) A schematic diagram showing the conserved binding site for miR-203. Putative conserved target sites in the LASP1 3'UTR were identified with the TargetScan database. One miR-203 target site is indicated. (B) LASP1 expression in ESCC cell lines after transfection of miR-203. Total-RNA was isolated after $48 \mathrm{~h}$ of transfection with miR-203. The mRNA expression levels of LASP1 were measured by TaqMan quantitative real-time PCR in TE2 and T.Tn cells. The results were normalized to the glyceraldehyde-3-phosphate dehydrogenase (GAPDH) expression and presented as the relative values compared to the control. "P<0.05. (C) The protein expression of LASP1 was analyzed by a western blot analysis $72 \mathrm{~h}$ after miR-203 transfection. The membranes were blotted with anti-LASP1 IgG and anti- $\beta$-actin IgG. $\beta$-actin was used as a loading control (the results were standardized to the levels of $\beta$-actin). (D) Total-RNA was isolated after $48 \mathrm{~h}$ of co-transfection with Syn-has-miR-203 miScript miRNA Mimic and miScript Target Protector/Negative control miScript Target Protector. The expression levels of LASP1 mRNA were measured by TaqMan quantitative real-time PCR. " $\mathrm{P}<0.05$.

also confirmed that the levels of the protein were remarkably decreased after $72 \mathrm{~h}$ (Fig. 3B). In the proliferation assay, we did not find any significant difference in cell growth inhibition between the si-LASP1-transfected cells and the control cells after $72 \mathrm{~h}$ (Fig. 4A) nor after 24, 48 or $96 \mathrm{~h}$ (data not shown), similar to the effects observed following the miR-203 treatment.

In the migration assay, we found a significant difference in the inhibition of cell motility between the si-LASP1-transfected cells and the control cells after $72 \mathrm{~h}(49.0 \%$ in TE2 and $80.6 \%$ in T.Tn) (Fig. 4B). Similarly, in the invasion assay, there was a significant difference in the inhibition of cell invasion between the si-LASP1-transfected cells and the control cells at $72 \mathrm{~h}$ after transfection (43.8\% in TE2 and 57.4\% in T.Tn) (Fig. 4C).

The relationship between $\mathrm{miR}-203$ and LASPI expression in ESCC clinical specimens. Total-RNA was isolated from matched non-cancerous esophageal epithelia and ESCC tissues, from which the miRNA and LASP1 mRNA levels were determined by TaqMan real-time PCR. In all 18 matched normal and cancer tissues, the expression levels of miR-203 were significantly lower in cancer tissues compared to noncancerous tissues (Fig. 5A). The correlation of the expression levels of LASP1 and miR-203 were tested using the Spearman rank correlation. There was a significant inverse correlation between the LASP1 mRNA and miR-203 expression levels $(\mathrm{P}=0.0398)$ (Fig. 5B).

The prognostic impact of miR-203 was further examined using the Kaplan-Meier method and the log-rank test. There was a significant correlation $(\mathrm{P}=0.0384)$ between patients with low miR-203 expression and a poor relapse free survival (RFS) (Fig. 5C).

\section{Discussion}

We have conducted searches for tumor suppressive miRNAs, such as miR-145, miR-133a, and miR-133b (20). In this study, we reported that miR-203 might directly control the expression of LASP1 and contribute to the inhibition of cell migration and invasion in ESCC. Since Yi et al mentioned 

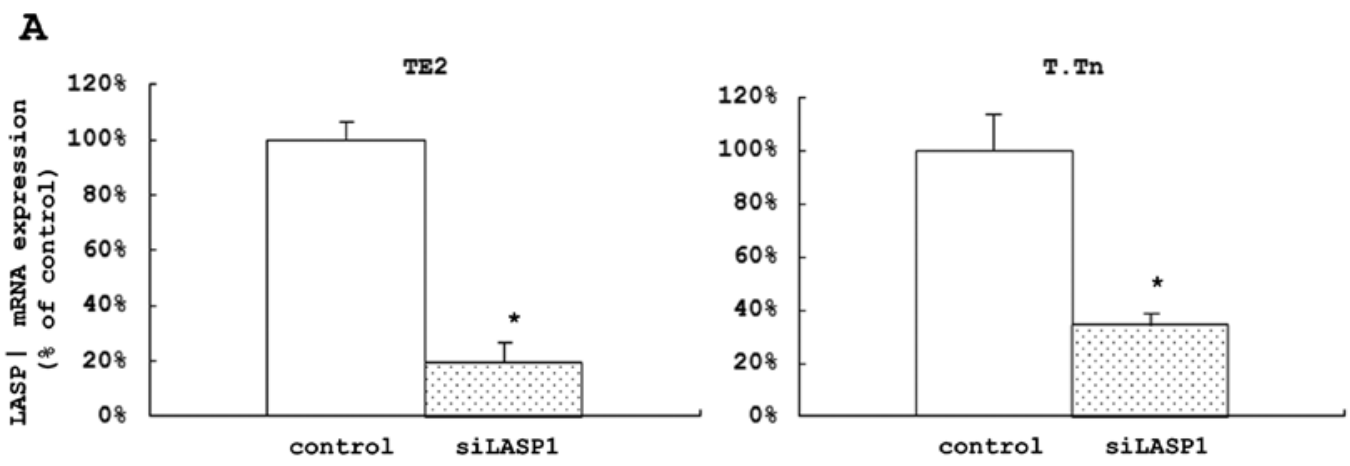

B
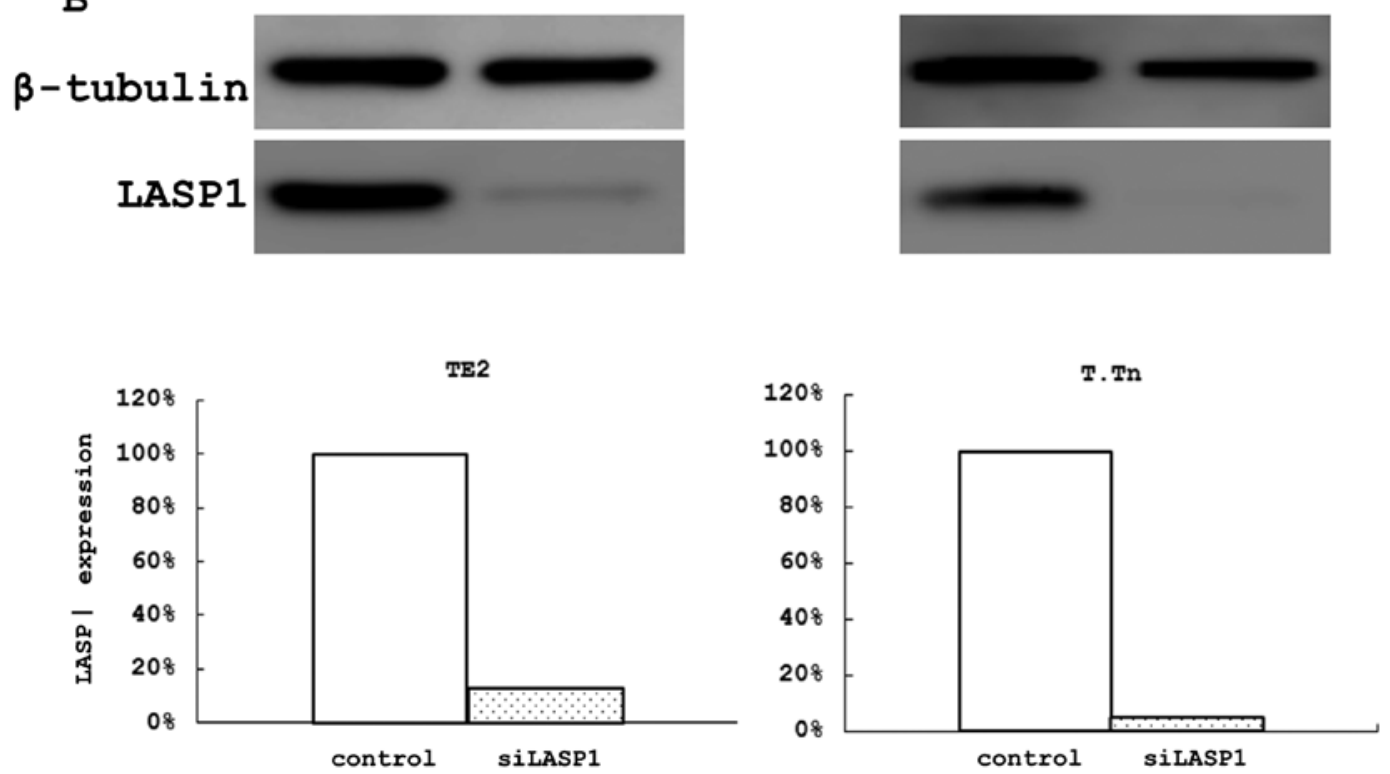

Figure 3. (A) si-LASP1 was transfected into ESCC cell lines (TE2 and T.Tn). After $48 \mathrm{~h}$ of transfection, the total-RNA was isolated and the expression levels were evaluated by TaqMan quantitative real-time PCR. "P $<0.05$. (B) After $72 \mathrm{~h}$ of transfection, cellular proteins were isolated and evaluated by a western blot analysis.

A

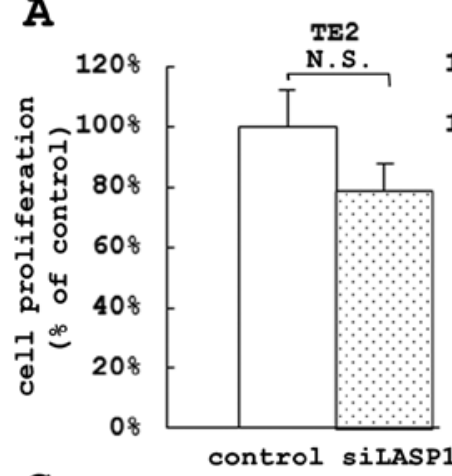

C

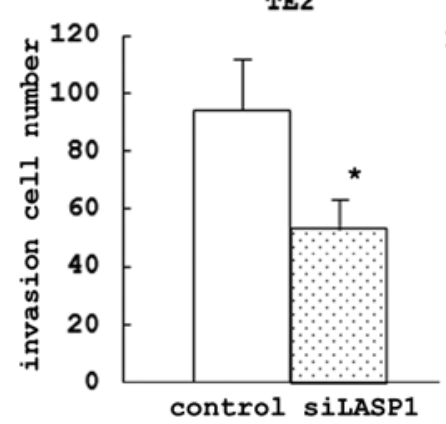

B

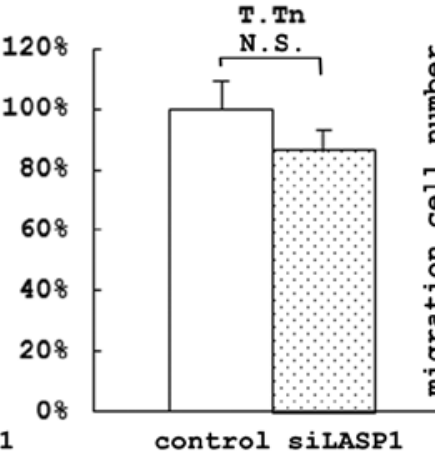

T. Tn

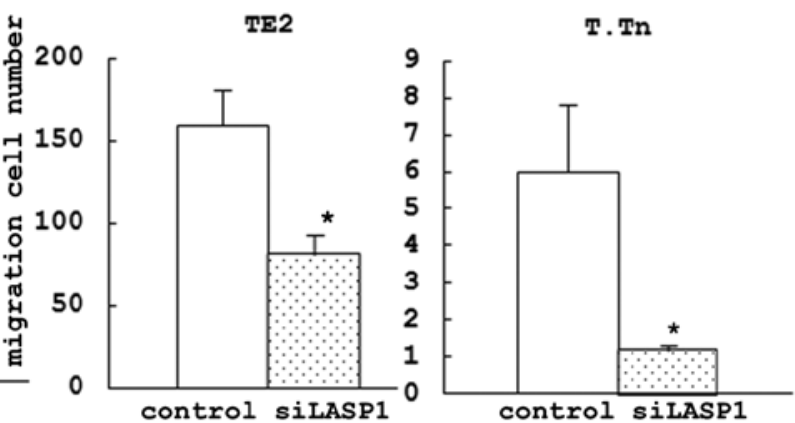

Figure 4. The effects of si-LASP1 on ESCC cell proliferation, migration and invasion. ESCC cell lines (TE2 and T.Tn) were transfected with si-LASP1 or negative control. Then, after $72 \mathrm{~h}$ of treatment were assessed for their (A) proliferation, (B) migration and (C) invasion compared to negative control cells. ${ }^{*} \mathrm{P}<0.05$. 
A

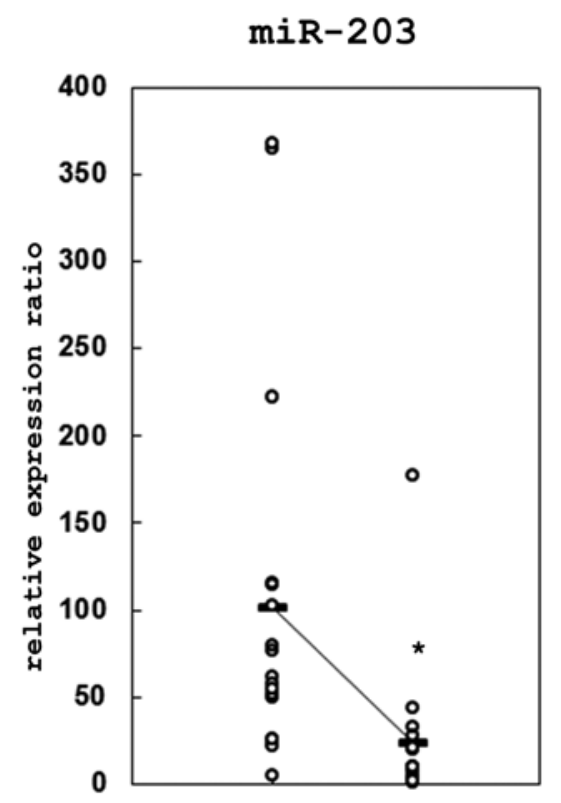

$\mathbf{N}$
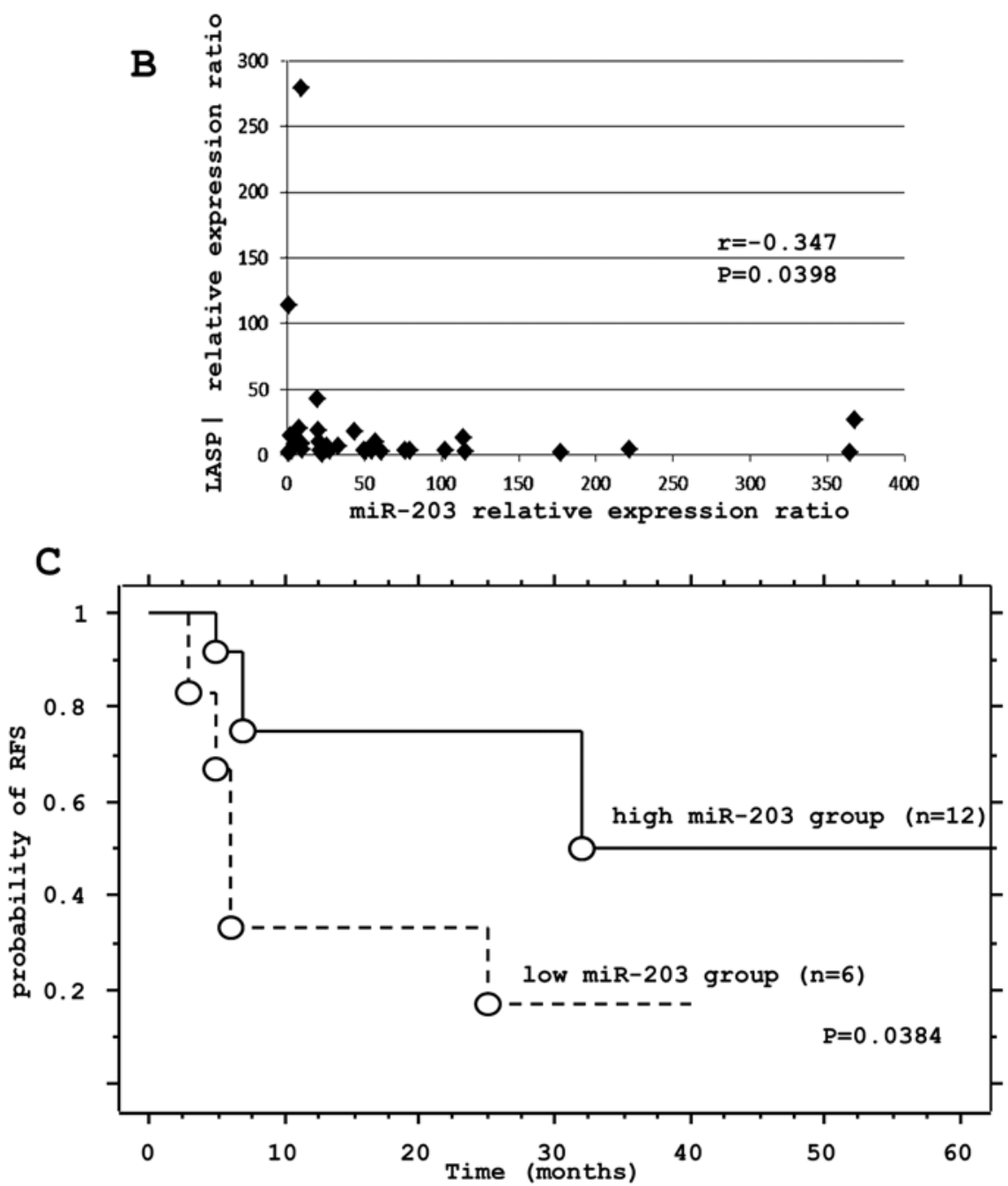

Figure 5. (A) The expression levels of miR-203 in ESCC clinical specimens. Total-RNA was isolated from 18 tumor tissues and matched noncancerous tissue samples using the TRIzol reagent. The expression levels of miR-203 were analyzed by TaqMan quantitative real-time PCR and normalized to the RNU6B expression. N, noncancerous tissues; C, cancer tissues. ${ }^{*} \mathrm{P}<0.05$. (B) The correlation between the LASP1 and miR-203 expression levels in ESCC clinical specimens. (C) The results of the Kaplan-Meier analysis of the relapse free survival (RFS) in patients with ESCC. The prognostic impact of miR-203 was further tested with the log-rank test.

that miR-203 might control epidermal differentiation by repressing p63 'stemness' (25), several studies have focused on the relationship between the expression of miR-203 and diverse malignancies. For example, it was suggested that miR-203 might play a crucial role in cell proliferation in head and neck squamous cell carcinomas, hematopoietic malignancies, and hepatocellular carcinoma (26-28). In ESCC, Feber et al initially reported that the expression of miR-203 was downregulated in Barrett esophagus and esophageal cancer compared to normal tissues (17). Bueno et al, Yuan et al and Bo et al revealed that miR-203 controlled cell proliferation in hematopoietic malignancies, bladder cancer, and ESCC by targeting ABL1, bcl-w and p63 (27,29,30). Moreover, other studies showed that miR-203 might be associated with not only cell proliferation, but also cell migration, cell invasion, and the epithelial-mesenchymal transition (EMT), by targeting ZEB2, Bmi, survivin and Runx2 (31,32).

In our study using two ESCC cell lines, TE2 and T.Tn, there was no relationship between miR-203 expression and cell proliferation. However, we found that the migration and invasion of both cell lines were significantly suppressed by miR-203. Our results also showed that the cell proliferation controlled by miR-203 in ESCC may depend on the individual cell line. In addition to these analyses, miR-203 is also considered to be a suppressor of cancer development, and Chen et al showed that higher expression of miR-203 might be useful as an independent predictor of a good prognosis (RFS and overall survival) of the patients with hepatocellular carcinoma and cirrhosis after liver transplantation (33). In our study, there was a significant correlation between lower expression of miR-203 in ESCC specimens and a poor RFS. Our result suggests that the expression of miR-203 could be a novel prognostic marker for patients with ESCC.

LASP1, known as one of the actin-binding proteins (molecular weight: $40 \mathrm{kDa}$ ), is localized to multiple sites of dynamic actin assembly, such as focal contacts, focal adhesions, lamellipodia membrane ruffles, and pseudopodia, and is involved in cell migration $(21,34,35)$. LASP1 was derived from a cDNA library of breast cancer metastases (21). This protein was highly overexpressed in $40 \%$ of metastatic 
human breast cancer tissues (21). Zhao et al reported that overexpression of LASP1 was also found in metastatic colorectal cancer tissues, and its higher expression was closely correlated with a poor prognosis of the patients with colorectal cancer (22). Therefore, it was suggested that LASP1 might have an important role in cancer cell mobility and metastasis, but the precise function of LASP1 in the various malignancies still remains unclear. No previous expression and functional analysis of LASP1 in ESCC has been reported. In our present study, there was no significant correlation between the expression of LASP1 and cell proliferation. On the other hand, the migration and invasion of ESCC cell lines were significantly suppressed by si-LASP1. Chiyomaru et al reported that miR-218, miR-1, and miR-133a regulated the expression of LASP1 in urinary bladder cancer (36), and Viticchiè $\mathrm{G}$ et al revealed that miR-203 regulated the expression of LASP1 in prostate cancer (37). Our study suggested that miR-203 and its target gene, LASP1, might be associated with the progression of ESCC.

Castilla et al reported that miR-203 was downregulated in the mesenchymal part of endometrial carcinosarcoma (31), and Saini et al mentioned that miR-203 regulated the EMT in prostate cancer (32). In this study, miR-203, which has been called an 'antimetastatic miRNA', was revealed to target LASP1 in ESCC, thus further strengthening its connection with cancer cell motility and metastasis.

The candidate target genes other than LASP1 were identified by other studies. Sadanandam et al reported that Semaphorin 5A (SEMA5A) was associated with tumor growth, invasion, and metastasis in pancreatic cancer (38), and Choi et al reported that overexpression of PDGFA associated protein 1 (PDAP1) was found in human rectal carcinoma (39). Song et al also reported that heparan sulfate 6-O-sulfotransferase 2 (HS6ST2) regulated the progression of pancreatic cancer by potentiating Notch signaling (40). Thus, there is a possibility that various pathways are affected by miR-203 in different cancers. We hope that the further analyses of the mechanism underlying the cancer progression regulated by miR-203 will contribute to the development of new cancer treatments and provide a new marker for the diagnosis of cancer in the future.

\section{References}

1. Chen J, Pan J, Zheng X, et al: Number and location of positive nodes, postoperative radiotherapy, and survival after esophagectomy with three-field lymph node dissection for thoracic esophageal squamous cell carcinoma. Int J Radiat Oncol Biol Phys 82: 475-482, 2012.

2. Yuequan J, Shifeng C and Bing Z: Prognostic factors and family history for survival of esophageal squamous cell carcinoma patients after surgery. Ann Thorac Surg 90: 908-913, 2010.

3. Altorki N, Kent M, Ferrara C and Port J: Three-field lymph node dissection for squamous cell and adenocarcinoma of the esophagus. Ann Surg 236: 177-183, 2002.

4. Medical Research Council Oesophageal Cancer Working Group: Surgical resection with or without preoperative chemotherapy in oesophageal cancer: a randomized controlled trial. Lancet 359: 1727-1733, 2002.

5. Blamey RW, Hornmark-Stenstam B, Ball G, et al: ONCOPOOL - a European database for 16,944 cases of breast cancer. Eur J Cancer 46: 56-71, 2010

6. Morris EJ, Sandin F, Lambert PC, et al: A population-based comparison of the survival of patients with colorectal cancer in England, Norway and Sweden between 1996 and 2004. Gut 60: 1087-1093, 2011
7. Ambros V: The functions of animal microRNAs. Nature 431: 350-355, 2004.

8. Bartel DP: MicroRNAs: genomics, biogenesis, mechanism, and function. Cell 116: 281-297, 2004.

9. He L and Hannon GJ: MicroRNAs: small RNAs with a big role gene regulation. Nat Rev Genet 5: 522-531, 2004.

10. Schickel R, Boyerinas B, Park SM and Peter ME: MicroRNAs: key players in the immune system, differentiation, tumorigenesis and cell death. Oncogene 27: 5959-5974, 2008.

11. He L, Thomson JM, Hemann MT, et al: A microRNA polycistron as a potential human oncogene. Nature 435: 828-833, 2005.

12. Calin GA, Trapasso F, Shimizu M, et al: Familial cancer associated with a polymorphism in ARLTS1. N Engl J Med 352: 1667-1676, 2005

13. Volinia S, Calin GA, Liu CG, et al: A microRNA expression signature of human solid tumors defines cancer gene targets. Proc Natl Acad Sci USA 103: 2257-2261, 2006.

14. Lu J, Getz G, Miska EA, et al: MicroRNA expression profiles classify human cancers. Nature 435: 834-838, 2005.

15. Iorio MV, Ferracin M, Liu CG, et al: MicroRNA gene expression deregulation in human breast cancer. Cancer Res 65: 7065-7070, 2005.

16. Yanaihara N, Caplen N, Bowman E, et al: Unique microRNA molecular profiles in lung cancer diagnosis and prognosis. Cancer Cell 9: 189-198, 2006.

17. Feber A, Xi L, Luketich JD, et al: MicroRNA expression profiles of esophageal cancer. J Thorac Cardiovasc Surg 135: 255-260, 2008

18. Guo Y, Chen Z, Zhang L, et al: Distinctive microRNA profiles relating to patient survival in esophageal squamous cell carcinoma. Cancer Res 68: 26-33, 2008.

19. Hiyoshi Y, Kamohara H, Karashima R, et al: MicroRNA-21 regulates the proliferation and invasion in esophageal squamous cell carcinoma. Clin Cancer Res 15: 1915-1922, 2009.

20. Kano M, Seki N, Kikkawa N, et al: miR-145, miR-133a and miR-133b: Tumor-suppressive miRNAs target FSCN1 in esophageal squamous cell carcinoma. Int J Cancer 127: 2804-2814, 2010.

21. Tomasetto C, Regnier C, Moog-Lutz C, et al: Identification of four novel human genes amplified and overexpressed in breast carcinoma and localized to the q11-21.3 region of chromosome 17. Genomics 28: 367-376, 1995.

22. Zhao L, Wang H, Liu C, et al: Promotion of colorectal cancer growth and metastasis by the LIM and SH3 domain protein 1. Gut 59: 1226-1235, 2010.

23. Takahashi K, Kanazawa H, Chan CT, Hosono T, Takahara M and Sato K: A case of esophageal carcinoma metastatic to the mandible and characterization of 2 cell lines (T.T and T. Tn) established from the oral tumor. Jpn J Oral Maxillofac Surg 36: 67-76, 1990.

24. Nishihira T, Kasai M, Mori S, et al: Characteristics of 2 cell lines (TE-1 and TE-2) derived from human squamous cell carcinoma of the esophagus. Gann 70: 575-584, 1979.

25. Yi R, Poy MN, Stoffel M and Fuchs E: A skin microRNA promotes differentiation by repressing 'stemness'. Nature 452: 225-229, 2008.

26. Lena AM, Shalom-Feuerstein R, Rivetti di Val Cervo P, et al: miR-203 represses 'stemness' by repressing DeltaNp63. Cell Death Differ 15: 1187-1195, 2008.

27. Bueno MJ, Pérez de Castro I, Gómez de Cedrón M, et al: Genetic and epigenetic silencing of microRNA-203 enhances ABL1 and BCR-ABL1 oncogene expression. Cancer Cell 13: 496-506, 2008.

28. Furuta M, Kozaki KI, Tanaka S, Arii S, Imoto I and Inazawa J: miR-124 and miR-203 are epigenetically silenced tumor-suppressive microRNAs in hepatocellular carcinoma. Carcinogenesis 31: 766-776, 2010.

29. Yuan Y, Zeng ZY, Liu XH, et al: MicroRNA-203 inhibits cell proliferation by repressing $\triangle \mathrm{Np} 63$ expression in human esophageal squamous cell carcinoma. BMC Cancer 11: 57, 2011.

30. Bo J, Yang G, Huo K, et al: microRNA-203 suppresses bladder cancer development by repressing bcl-w expression. FEBS J 278: 786-792, 2011.

31. Castilla MÁ, Moreno-Bueno G, Romero-Pérez L, et al: Micro-RNA signature of the epithelial-mesenchymal transition in endometrial carcinosarcoma. J Pathol 223: 72-80, 2011.

32. Saini S, Majid S, Yamamura S, et al: Regulatory role of mir-203 in prostate cancer progression and metastasis. Clin Cancer Res 17: 5287-5298, 2010. 
33. Chen HY, Han ZB, Fan JW, et al: miR-203 expression predicts outcome after liver transplantation for hepatocellular carcinoma in cirrhotic liver. Med Oncol: Jul 24, 2011 (Epub ahead of print).

34. Chew CS, Chen X, Parente JA Jr, Tarrer S, Okamoto C and Qin HY: Lasp-1 binds to non-muscle F-actin in vitro and is localized within multiple sites of dynamic actin assembly in vivo. J Cell Sci 115: 4787-4799, 2002.

35. Lin YH, Park ZY, Lin D, et al: Regulation of cell migration and survival by focal adhesion targeting of Lasp-1. J Cell Biol 165: 421-432, 2004

36. Chiyomaru T, Enokida H, Kawakami K, et al: Functional role of LASP1 in cell viability and its regulation by microRNAs in bladder cancer. Urol Oncol 30: 434-443, 2012.
37. Viticchiè G, Lena AM, Latina A, et al: miR-203 controls proliferation, migration and invasive potential of prostate cancer cell lines. Cell Cycle 10: 1121-1131, 2011.

38. Sadanandam A, Varney ML, Singh S, et al: High gene expression of semaphorin $5 \mathrm{~A}$ in pancreatic cancer is associated with tumor growth, invasion and metastasis. Int J Cancer 127: 1373-1383, 2010.

39. Choi SY, Jang JH and Kim KR: Analysis of differentially expressed genes in human rectal carcinoma using suppression subtractive hybridization. Clin Exp Med 11: 219-226, 2011.

40. Song K, Li Q, Peng YB, et al: Silencing of hHS6ST2 inhibits progression of pancreatic cancer through inhibition of Notch signalling. Biochem J 436: 271-282, 2011. 